\title{
Determinantes de la informalidad laboral en Colombia para el período $2008-2017$
}

\author{
Determinants of the labor informality in Colombia for the 2008 - 2017 period \\ Juan Esteban Berrio-Calle \\ Economista, Universidad de Medellín, Medellín-Colombia, jsberrio@hotmail.com \\ Lemy Bran-Piedrahita \\ Coordinador General de Investigaciones, Fundación Universitaria Católica del Norte, \\ Medellín-Colombia, Ibpiedrahita@ucn.edu.co
}

\begin{abstract}
Cómo citar / How to cite
Berrío-Calle, J. E., Bran-Piedrahita, L. (2019). Determinantes de la informalidad laboral en Colombia para el período 2008 - 2017. Revista CEA, 5(9), 33-51. https://doi.org/10.22430/24223182.1255

Recibido: 6 de agosto de 2018

Aceptado: 19 de diciembre de 2018

\section{Resumen}

El objetivo de esta investigación es identificar los determinantes clave que explican la informalidad laboral en Colombia, para el período 2008 - 2017. En el proceso se utilizó un enfoque metodológico cuantitativo, de tipo descriptivo, para ello se emplearon datos de la Gran Encuesta Integrada de Hogares $(\mathrm{GEIH})$, realizada por el Departamento Administrativo Nacional de Estadística (DANE), además de diversas fuentes referenciales de la literatura, donde los determinantes se catalogaron como cíclicos y acíclicos, dependiendo de su comportamiento, respecto al ciclo económico. Los análisis arrojan que Bogotá, Medellín, Manizales, Pereira y Cali, presentan las tasas de informalidad más bajas (entre $40 \%$ y $50 \%$ ). Adicionalmente, entre las conclusiones resalta que existe una relación lineal o directa entre la duración del desempleo y la informalidad; a mayor tiempo en calidad de desempleado, mayor probabilidad de caer en la informalidad.
\end{abstract}

Palabras clave: ciclo económico, desempleo cíclico, desigualdad social, sector informal, sistema económico.

\begin{abstract}
The objective of this study is to identify the key drivers that explain labor informality in Colombia in the period from 2008 to 2017. A quantitative descriptive method was adopted to analyze data from the Great Integrated Household Survey $(\mathrm{GEIH})$ conducted by the National Administrative Department of Statistics (DANE) as well as several important sources in the literature. Drivers were classified into cyclic and acyclic, depending on their behavior with respect to the general economic cycle. The analysis revealed that Bogotá, Medellín, Manizales, Pereira, and Cali present the lowest rates of informal employment in the country (between $40 \%$ and $50 \%$ ). Additionally, the most
\end{abstract}


outstanding conclusion is that there is a linear or direct relationship between the length of unemployment and informality: the longer individuals are unemployed, the more likely they are to join the informal sector.

Keywords: economic cycle, cyclical unemployment, social inequality, informal sector, economic system.

\section{INTRODUCCIÓN}

El fenómeno de la informalidad es una manifestación del mercado laboral que se ha presentado principalmente en países en vía de desarrollo, además de ser un problema latente en países de América Latina, tales como Colombia, Brasil, Venezuela y México. Este problema se origina como producto del desempleo y debe su extensión, en parte, a la globalización económica; esto se ha traducido en la deslocalización de procesos de producción, lo cual contribuye en gran medida a perpetuar la economía informal (Portes y Haller, 2004). El empleo informal está caracterizado por darse en unidades de producción a pequeña escala, con baja productividad, empleo poco estable, ingresos escasos, volátiles y con acceso bajo o nulo a los sistemas de protección social contributiva (Centro de Estudios del Trabajo -Cedetrabajo-, 2016).

Uno de los dos principales enfoques teóricos que explican la informalidad laboral es el enfoque estructuralista de corte macroeconómico, en el cual la informalidad surge por la necesidad de supervivencia; es decir, es una alternativa que se origina como respuesta a la dificultad que existe de ingresar al mercado laboral y de obtener un empleo de calidad en un sector moderno reducido, en el que la demanda solo capta a los candidatos más aptos, dados sus niveles de cualificación (Jiménez, 2012).

Por otro lado, en el enfoque institucionalista de corte microeconómico, el sector informal está caracterizado por agrupar actividades económicas de carácter legal, pero que, debido al incumplimiento de reglamentaciones comerciales, laborales y tributarias, se consideran ilegales (Jiménez, 2012). Esta visión también teoriza el cómo las instituciones incentivan a los agentes económicos a no aceptar la normatividad y preferir desarrollar sus actividades económicas en condición de informales. Finalmente, existe un tercer enfoque mixto, con aportes de los dos enfoques (estructuralista e institucionalista), en donde la estructura económica y productiva de un país y la elección racional de los agentes económicos son los factores que explican el origen de la informalidad (Jiménez, 2012). En el caso de Colombia, el Departamento Administrativo Nacional de Estadística (DANE) mide la informalidad de acuerdo con la visión institucionalista, y cuenta en la población informal a los individuos que no coticen en los sistemas sociales de salud y pensiones (DANE, 2009).

América Latina ha experimentado cambios significativos, entre los que se destacan: un crecimiento del Producto Interno Bruto -PIB- per cápita, entre los años 2000 y 2013, en cerca de un 30.4 \%; el egreso de más de 50 millones de personas que estaban en condiciones de pobreza, y que comienzan a constituirse en una nueva clase media emergente; y el auge de las exportaciones, favoreciendo las estructuras económicas de los países de la región. Empero, persisten las desigualdades sociales, siendo la zona más desigual del mundo (Rivas y Sánchez, 2016). 
En este orden de ideas, es importante aclarar que la informalidad se vincula con empleos poco estables, donde no es necesaria la alta cualificación del talento humano, y suelen realizarse actividades por organizaciones pequeñas; de hecho, la inestabilidad laboral es un factor común, reflejado en condiciones de trabajo precarias y la omisión de aportes para seguridad social; lo anterior, menoscaba la calidad de vida de las personas y, por tanto, hace más recalcitrante la pobreza y la exclusión social (Quejada, Yánez y Cano, 2014; Carvajal, Cárdenas y Estrada, 2017).

Para Colombia, el caso es similar al resto de la región, pero deben considerarse fenómenos que contribuyen el agravamiento de la informalidad, como el conflicto armado, con diversos grupos al margen de la ley como los Paramilitares, las desmovilizadas Fuerzas Armadas Revolucionarias de Colombia (FARC), el Ejército de Liberación Nacional (ELN), el también desmovilizado Movimiento 19 de Abril (M-19), las bandas criminales y otros; derivado, entre otras causas, por las formas de tenencia y asignación de la tierra y otros agravios que configuran su prolongación (Berry, 2002; Palacios, 2012; González, 2014). Estos grupos actúan principalmente en las zonas rurales del país, privando a familias de sus medios de subsistencia, perjudicando el sector agropecuario de la economía y desplazando una masa laboral sin demanda hacia las zonas urbanas.

Por tanto, analizar la informalidad laboral del país demanda comprender estas dinámicas, que se configuran con otras variables sociales, políticas y culturales en una problemática compleja de intervenir. Al respecto, el abordaje de este fenómeno precisa considerar el comportamiento asimétrico entre una región y otra -dada la diversidad no solo cultural, sino también económica y social existente-; por lo cual, los ejercicios de medición de esta problemática, han estado liderados en el país por el DANE, según directrices de la Organización Internacional del Trabajo -OIT- (Mora, 2017; Arango y Flórez, 2017).

Así, en el año 2012 es aprobada en el país una reforma tributaria, la cual entre sus principales justificaciones tenía el incentivar la formalización laboral. Sin embargo, de acuerdo con las estimaciones develadas por el Plan Nacional de Desarrollo 2014 - 2018, si se efectuara la medición de informalidad en Colombia, teniendo como base la ausencia de registro mercantil y contabilidad, existiría un $43 \%$ de organizaciones sin dicho registro y un 61 \% que no llevan a cabo ejercicios contables en rigor (Osorio, 2016; Vergara, Ramírez, Naranjo y Carranza, 2017). Por lo anterior, se propuso la realización de un estudio que permitiera identificar los determinantes clave que explican este fenómeno en el país.

\section{MARCO TEÓRICO}

Las condiciones del mercado laboral se constituyen en una temática de interés prioritario en las agendas políticas de los países, especialmente de aquellos en vías de desarrollo -como los latinoamericanos-, donde analizar la relación existente entre el desempleo y la informalidad otorga un espectro clave para comprender las dinámicas de dicho mercado; el primero devela la cantidad de desocupados en un territorio, y la segunda, hace referencia a elementos de calidad, como la productividad y la adherencia al marco normativo aplicable (Jiménez, 2012; Ramírez, Zambrano, Mogrovejo y Carreño, 2016). 
Por ello, en Latinoamérica, las tasas de informalidad laboral son un asunto prioritario para los hacedores de política pública, dado el impacto de este problema en los lineamientos sociales y económicos que emanan desde los Gobiernos. Además, aunque comenzando el siglo XXI los niveles de informalidad presentaron reducciones en la región, continúan siendo elevados -cercanos al 50 \%- (Jiménez, 2012; Levy y Székely, 2016), lo cual impacta las posibilidades de crecimiento de las economías y las dinámicas de desarrollo.

En Colombia, acorde con las dinámicas regionales, la informalidad laboral se ha convertido en una problemática de alta relevancia, donde sus repercusiones involucran el Sistema de Seguridad Social Integral, respecto a las contribuciones de la población trabajadora al sistema pensional, pues la alta informalidad del mercado laboral del país conlleva a un desequilibrio en este renglón; además, este fenómeno ha afectado también el nivel de ingreso de la población y, por consiguiente, su calidad de vida, tal como lo expone Galvis (2012) en su estudio.

De hecho, tal como señalan González y Daza (2015), en el país se emplean diversos instrumentos para monitorear este fenómeno, entre ellos las encuestas de hogares -como es el caso de la Gran Encuesta Integrada de Hogares (GEIH)-, las cuales permiten conocer con mayor detalle la situación laboral de las personas -en función de variables como el ingreso y la garantía de cotizar al sistema de seguridad social-, así como caracterizar la población; encuesta sobre la cual se han fundamentado estudios de diferentes académicos, como el de Carvajal, et al. (2017) sobre la informalidad laboral en el área metropolitana de Bucaramanga.

Así, el interés por estudiar el comportamiento de este fenómeno en Colombia ha llevado a encontrar riqueza de información, no solo en fuentes oficiales -como la GEIH-, sino también en la literatura científica, entre ellas la propuesta por Quejada, et al. (2014), quienes a través de un estado del arte exponen la incidencia que han tenido la globalización, la política económica y el desarrollo industrial en la informalidad, donde solo por citar un ejemplo, un crecimiento menos acelerado de la industria puede llevar a incrementar los niveles de informalidad en el mercado laboral.

De otro lado, la investigación realizada por García (2012) expone otras aristas de este fenómeno, al revelar el papel clave que ejerce la educación de la población ocupada en la reducción de su propensión a la informalidad, dado que cada año de formación del individuo reduce hasta en un $4 \%$ la probabilidad de emplearse informalmente; además, refiere García (2012), la influencia de factores propios de cada ciudad colombiana, que pueden afectar el comportamiento de la informalidad laboral, como son la ubicación geográfica de un territorio, la integración comercial y la composición de los diversos sectores económicos.

En adición, autores como Castro (2014), presentan un análisis riguroso frente a la informalidad laboral en Colombia, pero hace hincapié sobre otros elementos que deben ser considerados al estudiar este problema, como es el rol del Estado y sus instituciones, quienes propician la vinculación al mercado informal por parte de las personas, al carecer de apropiados instrumentos de control para asegurar el cumplimiento de la norma, en lo que atañe a la garantía de derechos mínimos tipificados en las diferentes legislaciones existentes en el país; además, el autor alude también la propensión de las mujeres para caer en la informalidad, por las presiones que reciben en ser productivas laboralmente -generar ingresos- y a su vez, cumplir con el rol ejercido tradicionalmente en la familia. 
Por su parte, el estudio de Salinas, González y Marín (2012), corrobora el planteamiento de Castro (2014), al señalar que las mujeres tienen mayor probabilidad de ingresar en la informalidad, de hecho, de acuerdo con los autores, son estas y los trabajadores por cuenta propia quienes constituyen mayoritariamente el mercado laboral informal en el país; pero también expone cómo se reduce el riesgo de ser parte de este mercado con la edad, el nivel educativo y el estar casado.

Desde otras aproximaciones encontradas en la literatura, llama la atención la investigación realizada por Osorio (2016), quien a través de un modelo general dinámico y estocástico, evaluó el impacto que tuvo para este fenómeno la reforma tributaria implementada en el país en 2012, encontrando así que entre los años 2012 y 2014 la tasa de informalidad disminuyó en 2,3 puntos porcentuales, lo que permite encontrar análisis favorables para esta problemática y que de hecho, pueden servir como referente para futuras investigaciones.

Es por ello que el panorama expuesto lleva a inferir la importancia que tiene esta temática en las agendas investigativas de las Instituciones de Educación Superior -IES- del país, de forma consecuente con las dinámicas nacionales; y a su vez devela la relevancia de continuar aportando al campo de conocimiento.

\section{METOdOLOGÍA}

Se efectuó un estudio con enfoque cuantitativo, de tipo descriptivo, cuyo objetivo consistió en identificar los determinantes clave que explican la informalidad laboral en Colombia, para el período 2008-2017. Para ello, se empleó la GEIH (2018) (anteriormente denominada Encuesta Continua de Hogares -ECH-), compilada por el DANE, midiendo temáticas de interés nacional como mercado laboral, salud, educación, justicia y trabajo infantil (Barraza, 2010).

Esta tiene cobertura nacional, que abarca zonas urbanas y rurales de las 13 ciudades principales, con sus áreas metropolitanas, y 11 ciudades intermedias; lo anterior, confiere validez a la información arrojada, y, por tanto, confiabilidad para el ejercicio de recolección y análisis de los datos (Gran Encuesta Integrada de Hogares, 2018). El análisis se realizó con datos de las 13 ciudades principales y sus áreas metropolitanas, que corresponden a: i) Bogotá, ii) Medellín (Valle de Aburrá), iii) CaliYumbo, iv) Barranquilla-Soledad, v) Bucaramanga-Floridablanca-Girón-Piedecuesta, vi) ManizalesVillamaría, vii) Pasto, viii) Pereira - Dosquebradas - La Virginia, ix) Ibagué, x) Cúcuta-Villa del RosarioLos Patios-El Zulia, xi) Villavicencio, xii) Montería, y xiii) Cartagena.

De otro lado, los datos se importaron al paquete estadístico Stata; además, teniendo en cuenta la metodología utilizada por el DANE para medir la informalidad, el tratamiento de datos y la construcción de los indicadores de tasa de informalidad se realizaron de acuerdo con el enfoque institucionalista, en el cual la población informal está constituida por los individuos de la población ocupada que no cotizan a los sistemas de seguridad social (salud y pensiones). Teniendo en cuenta lo anterior, fue posible analizar la informalidad laboral, a través de determinantes cíclicos (tasa de desempleo, tasa de interés e inversión y comercio exterior) y acíclicos (nivel educativo, rama de actividad, ciudad, costos de transacción y barreras de entrada, así como la edad y el sistema de pensiones). 
Para clasificar los determinantes de la informalidad (cíclicos y acíclicos), se tomó como referencia el trabajo de Rabanal (2014, como se citó en Gómez, 2007), quien se apoya en los aportes de Friedrick von Hayek, en cuanto a la estructura del ciclo económico, elementos a través de los cuales fue posible llevar a cabo el análisis de los datos recolectados.

\section{RESULTADOS Y DISCUSIÓN}

Los determinantes cíclicos son aquellos que dependen del ciclo económico, y que varían, sujetos a la etapa en que se encuentre la economía; en cuanto a los determinantes acíclicos, son variables que no supeditan su comportamiento al estado del ciclo económico, aunque este influye en su comportamiento de manera indirecta. A continuación, se presenta la forma en que se estructuró dicho análisis:

\section{Período 2008 - 2017 de la Gran encuesta integrada de hogares}

Colombia es la sexta economía con mayor informalidad en América Latina, región con las tasas más altas de empleo informal, inclusive por encima de países de África Subsahariana (Casabón, 2017). Por ello, el Gobierno colombiano ha realizado diferentes avances para frenar la informalidad y favorecer el crecimiento económico, con el objetivo de aumentar la base tributaria, mediante el uso de estrategias legales y políticas; no obstante, la inexistencia de procesos de evaluación ha limitado el conocimiento del impacto de estas acciones (Consejo Privado de Competitividad, 2017).

Teniendo en cuenta lo anterior, se consideró pertinente la realización de este estudio, en tanto permitió identificar los factores que inciden en el fenómeno de la informalidad laboral. Así, tal como se expone en la Figura 1, se pudo sustraer de la GEIH la información sobre la tasa de informalidad entre el período 2008 a 2017:

A finales de los años noventa y en los albores del año 2000, la tasa de informalidad era cercana al 60 $\%$. En el periodo observado (2008 - 2017) se puede apreciar una tasa aproximada del 51,23\% para el año 2008 , la cual se mantiene relativamente estable (entre $50 \%$ y $52 \%$ ), comenzando un descenso progresivo desde el 2012, hasta llegar a 47,56 \% en el tercer trimestre del año 2017.

\section{Determinantes cíclicos de la informalidad laboral}

\section{Desempleo}

Desde 2004, la tasa de desempleo registra un descenso por debajo del $12 \%$, hasta el año 2008 , lo cual se explica por un buen desempeño económico y una tasa de informalidad que se incrementa en el periodo 2006 - 2008. Entre el año 2008 y 2010, la tasa de desempleo refleja un leve incremento, tal como se ve en la figura 2, que puede obedecer al período de la crisis hipotecaria que inició en 2008, y cuyas repercusiones afectaron la mayor parte de las economías mundiales; esta empieza a experimentar una reducción sostenida por debajo del $10 \%$, a partir del año 2013, aunado con una disminución en la tasa de informalidad a partir del mismo periodo (por debajo del $50 \%$ ), como se observa en la Figura 1. Esto se traduce en un aumento al acceso de trabajo digno y formal, así como mejores condiciones laborales y un sector productivo con mayor capacidad de empleabilidad. 


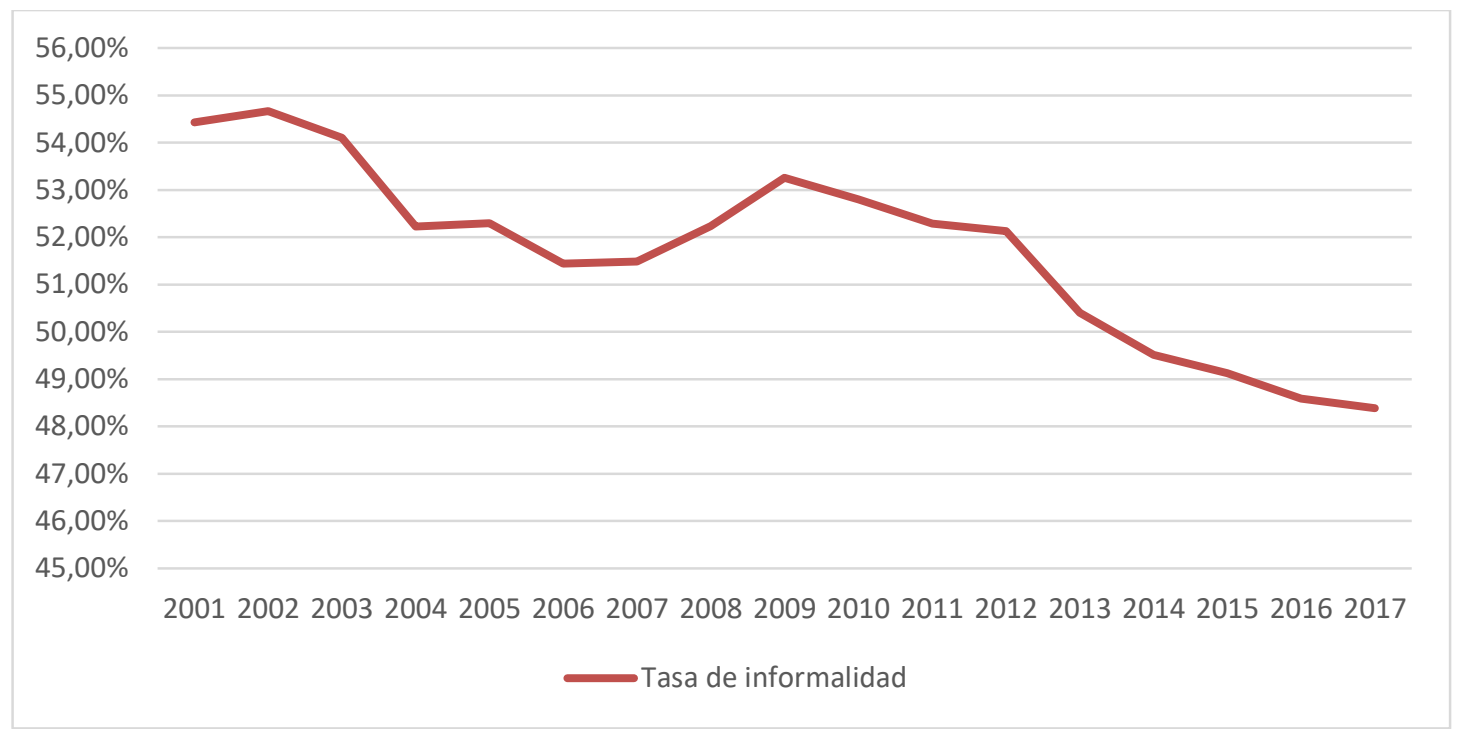

Figura 1. Evolución de la tasa de informalidad en el período (2001 - 2017)

Figure 1. Evolution of the informality rate (2001-2017)

Fuente: elaboración de los autores con datos de la Gran Encuesta Integrada de Hogares del DANE, 2017-2018.

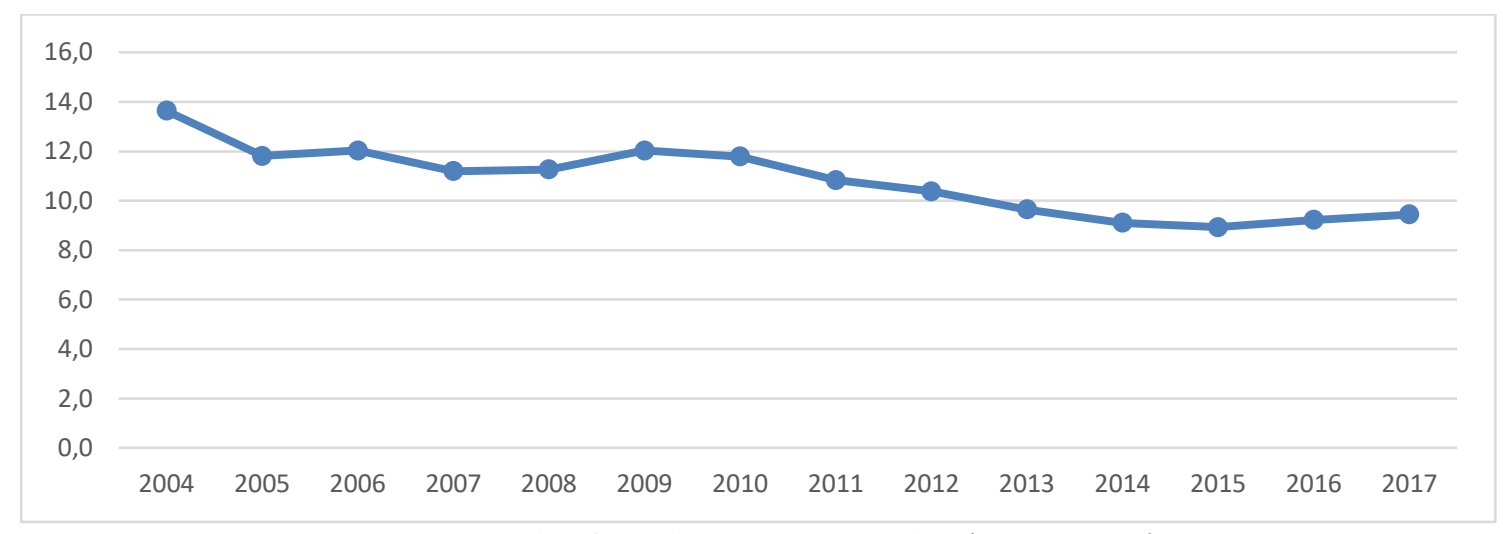

Figura 2. Evolución de la tasa de desempleo (2004 - 2017)

Figure 2. Evolution of the unemployment rate $(2004-2017)$

Fuente: elaboración de los autores con datos de la Gran Encuesta Integrada de Hogares del DANE, 2017-2018.

Los resultados obtenidos por Marcillo (2010), en un estudio con datos de la GEIH, evidencian que los individuos con una mayor duración en estado de desempleo, tienen más probabilidad de ser informales. Con base en este planteamiento, se infiere que la relación entre la duración del desempleo y la probabilidad de ser informal es de tipo directa, es decir, entre más prolongado es el tiempo de acceder a un empleo formal, mayor probabilidad existe, para un individuo, de ingresar a la informalidad laboral. Con base en lo anterior, se establecen tres hipótesis por las cuales la tasa de desempleo es causante de informalidad.

La primera razón está relacionada con la falta de una institución que se encargue de conectar el mercado laboral; a pesar de que existen bolsas de trabajo y puestos de gobiernos departamentales que ayudan a la gente a buscar empleo, muchos no usan este sistema; además, los que están 
establecidos no cuentan con la suficiente legitimidad otorgada por la masa laboral, debido a la percepción negativa. Lo anterior, se puede explicar, en tanto la mayor parte de las personas que usan este tipo de sistemas tienen niveles educativos bajos y medios, lo que ocasiona una falta de confianza en el sistema, al no poder encontrar demanda laboral para ellos; así las cosas, esto se traduce en un aumento de la duración del desempleo, mientras el trabajador se adapta o cualifica para cumplir los requisitos del mercado laboral.

La segunda, estaría relacionada con el nivel educativo, ya que este, como se describió anteriormente, influye significativamente a la hora de conseguir trabajo; en este orden de ideas, si la persona no cumple con los requerimientos del mercado laboral, ya sea porque tiene baja educación o porque la carrera de elección no tiene campo laboral en Colombia, entonces no podrá buscar trabajo hasta que se adapte a los mismos, así sea porque encuentre un empleo en su área de conocimiento o porque diversifica dichos conocimientos para tener un campo laboral más amplio; finalmente, el tiempo que se demore en suceder alguna de las dos situaciones descritas contribuye a generar informalidad, ya que la persona se verá obligada a incurrir en otras actividades, en aras de subsistir, y estas pueden ser informales.

Finalmente, se tiene el desempleo generado por el ciclo económico; este se genera cuando entra en crisis la economía, en tanto las empresas despiden trabajadores, se afectan las industrias y las personas quedan desempleadas, por lo que, obligadas a sobrevivir, entran en la economía informal.

\section{Tasa de interés e inversión}

La Figura 3 muestra el diferencial de tasa de interés entre varios países; como puede observarse, para el caso de Colombia, este indicador es de los más altos, y el segundo más alto, por debajo de Uruguay (aunque para el periodo 2010 - 2012 fue mayor el de Colombia). Se observa, también, que países de la región como México y Chile tienen unos diferenciales en tasa más bajos, cercanos a los de países como Suiza y China. El hecho de que Colombia tenga un diferencial tan alto puede ser un factor contribuyente a la alta tasa de informalidad (debido a los factores explicados anteriormente), y más aun teniendo en cuenta la vasta desigualdad en tecnología y capital entre los dos sectores, formal e informal (lo cual sería explicado por una tasa de interés alta).

La relación entre la tasa de interés, la inversión y la informalidad queda clara en un concepto económico planteado por la escuela clásica. Véliz y Díaz (2014) comentan que la creación de la riqueza parte del ahorro, el cual se transforma en inversión mediante un proceso denominado Ley de Preferencia Temporal, para después convertirse en bienes de capital que contribuyen, de manera decisiva, al aumento de la productividad y la generación de producción, fomentando así el bienestar social. 


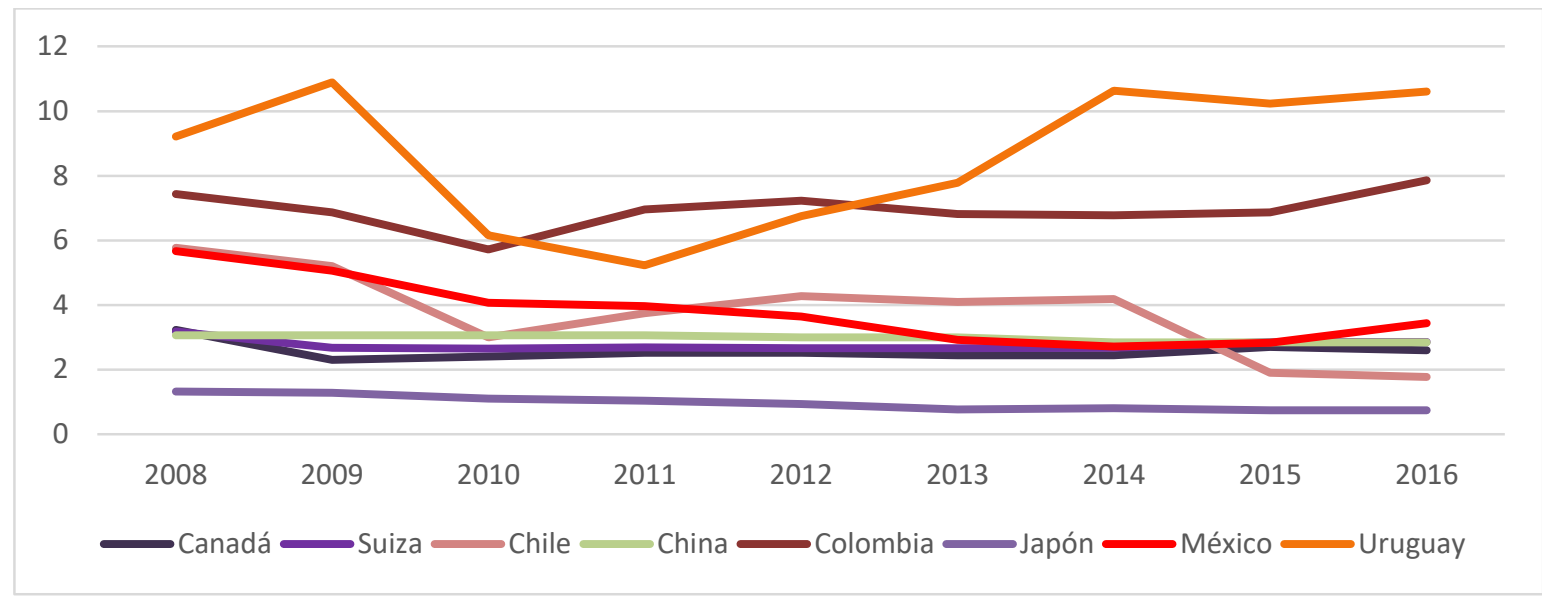

Figura 3. Diferencial de tasas de interés $(2008$ - 2016) y Evolución de la tasa de desempleo (2004 - 2017)

Figure 3. Interest rate differentials (2008-2016) and evolution of the unemployment rate (2004-2017)

Fuente: elaboración de los autores con datos del Banco Mundial.

Si existe ahorro en una economía, se puede realizar inversión en capital y tecnología para aumentar la productividad, lo cual incrementa la producción y mejora el consumo y los salarios; esto se traduce en ahorro por parte de los agentes económicos, y contribuye a generar un ciclo. Adicionalmente, una tasa de interés que sea baja permite una mayor demanda de dinero, contribuyendo a la inversión y modernización de procesos productivos, para así incrementar competitividad y productividad.

El conflicto surge cuando existen altas tasas de interés que direccionan la inversión extranjera a grandes empresas del sector formal, $y$, en contraste, dejan a las pequeñas unidades de trabajo o empresariales sin la oportunidad de modernizar tecnología, procesos y capital humano, debido al alto costo del dinero, estancando de esta manera los agentes que operen en informalidad, al caracterizarse este sector por baja productividad, uso de herramientas rudimentarias y evasión de sistemas tributarios y sociales.

\section{Comercio exterior}

La evidencia empírica devela que no siempre el comercio mundial genera informalidad, pero si es claro que, para el caso de Colombia, la apertura económica y el posterior desarrollo del comercio exterior del país, si tuvo un efecto en el aumento de la informalidad, teniendo en cuenta que desde el año 2013 la balanza comercial viene incrementando una brecha negativa; entonces, es fácil abstraer de este indicador que las importaciones realizadas por los colombianos son mayores a lo que se exporta, y donde muchas de esas importaciones son productos intensivos en mano de obra que compiten con las industrias ya establecidas en el país, lo cual desplaza trabajadores y genera informalidad como vía de escape. En la Figura 4 puede apreciarse el comportamiento de estas dos variables de la balanza comercial, en el período 2000-2017, y donde, desde el año 2013, se refleja un punto de quiebre que ha mostrado la brecha entre las importaciones y exportaciones del país.

Esta temática de cómo la integración a una economía de intercambio global afecta la informalidad en países con economías en desarrollo es tratada por la Organización Mundial del Comercio (2009), a partir de los resultados obtenidos en los primeros estudios realizados, donde se observó que la apertura económica y el comercio entre países trae informalidad para una de las partes; esto porque 
el sector formal tiene que competir con importaciones baratas, lo cual típicamente empuja a los trabajadores hacia el sector informal; adicionalmente, dependiendo de la movilidad del capital y los vínculos de producción, puede llevar a un aumento o reducción de los salarios informales.

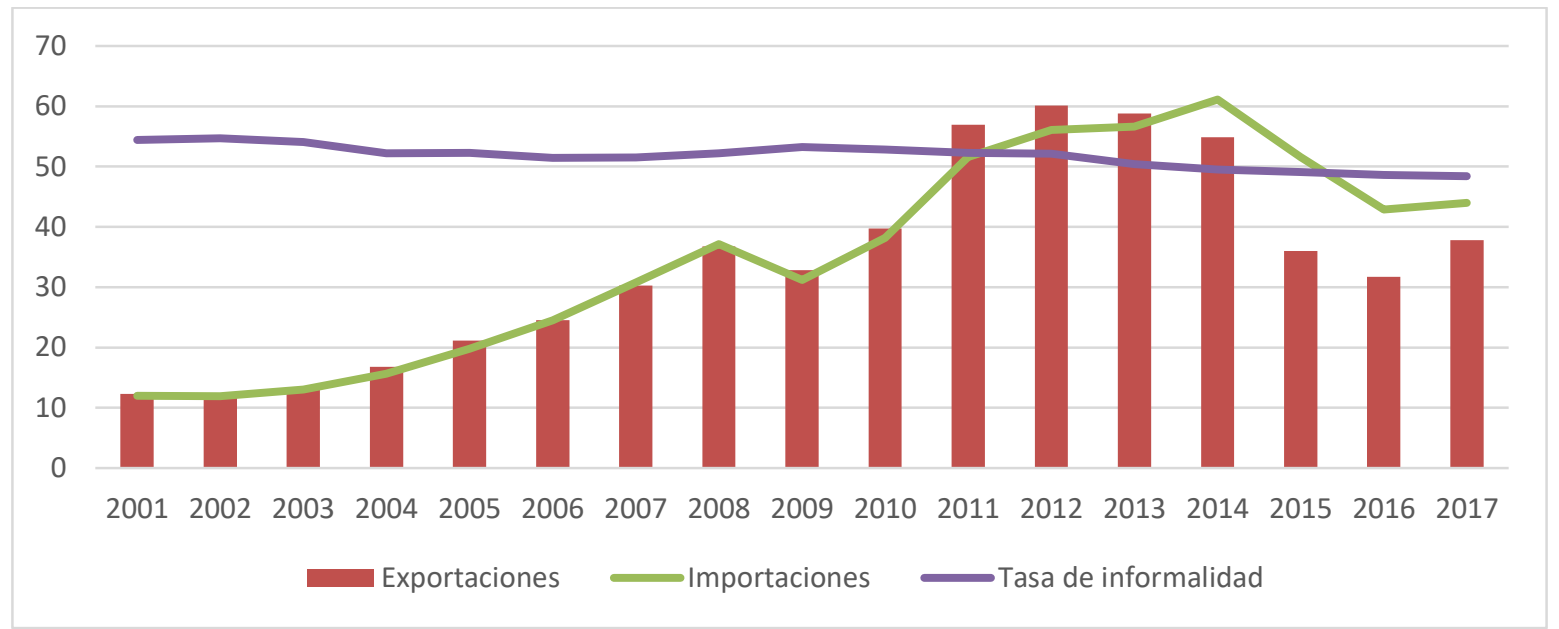

Figura 4. Balanza Comercial y tasa de informalidad para Colombia (2001 - 2017)

Figure 4. Balance of trade and informality rate in Colombia (2001-2017)

Fuente: elaboración de los autores con datos del Banco Mundial y la Gran Encuesta Integrada de Hogares del DANE, s.f.

En Colombia, las exportaciones se componen principalmente por materias primas y algunos productos intensivos en mano de obra, y con importaciones de productos intensivos en mano de obra y capital, donde generalmente se produce una balanza comercial negativa. Por el contrario, en economías desarrolladas se exportan productos intensivos en capital, se importan materias primas, y el resultado es una balanza comercial positiva (Simoes e Hidalgo, 2011). Al respecto, la Organización Internacional del Trabajo y la Organización Mundial del Comercio (2009) disertan señalando que la apertura económica y el comercio entre países trae informalidad para una de las partes, debido a la introducción de un ambiente competitivo, lo que puede desplazar trabajadores hacia el sector informal; en adición, y dependiendo de la movilidad del capital y los vínculos de producción, puede llevar a un aumento o reducción en salarios.

\section{Determinantes acíclicos de la informalidad laboral}

\section{Nivel educativo}

La Figura 5 revela la tasa de informalidad según el nivel educativo de la persona; aquellas sin ningún nivel educativo y con básica primaria, presentan la tasa más alta - con más del $80 \%$-, seguido por personas con educación secundaria -cerca de un $60 \%$-, y finalmente, individuos con un nivel superior de educación - cuyo registro es cercano al 30 \% -. Las tasas son estables a través del tiempo, y la evidencia refleja que el nivel de educación tiene una relación inversa con la probabilidad de ser informal: a mayor nivel de educación, menor es la probabilidad de incurrir en la economía informal. 


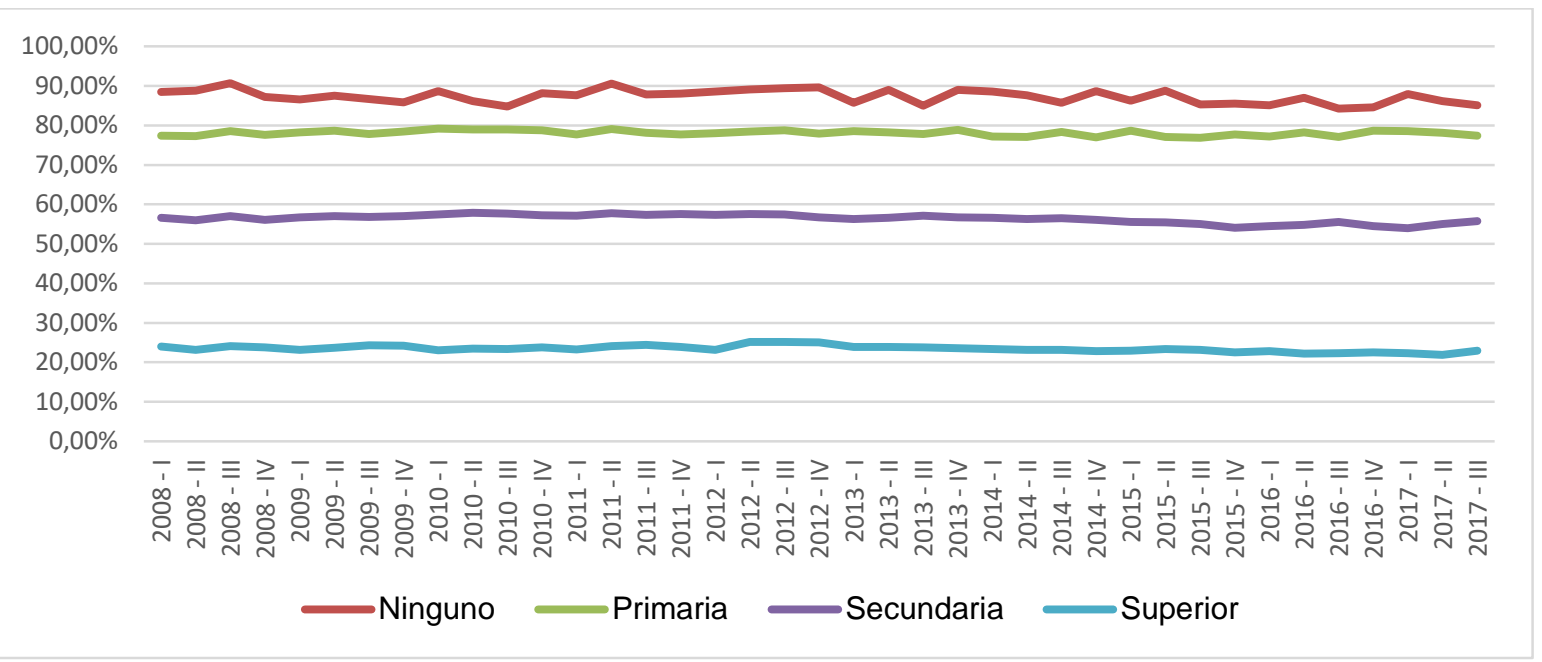

Figura 5. Tasa de informalidad por nivel educativo en el período 2008 - 2017 y evolución de la tasa de informalidad en el período 2008 - 2017

Figure 5. Informality rate by educational attainment (2008-2017) and evolution of the informal employment rate (2008-2017)

Fuente: elaboración de los autores con datos de la Gran Encuesta Integrada de Hogares del DANE, 2017-2018)

La anterior afirmación es validada por autores como Barco y Vargas (2010), quienes aseveran que contar con una educación secundaria y superior incrementa la probabilidad de tener ingresos provenientes de un empleo formal; lo que permite colegir que la educación es un determinante acíclico de la informalidad laboral. Sin embargo, es pertinente, de cara a este ejercicio, contemplar futuros estudios que profundicen en asuntos medulares, asociados a la calidad de la formación ofrecida a las personas que pueden acceder a ella, y cómo ésta puede incidir en la propensión a la informalidad.

\section{Rama de actividad}

De acuerdo con los datos obtenidos de la GEIH, los sectores en los cuales se registra mayor informalidad son: i) construcción, ii) comercio, hoteles y restaurantes, iii) transporte, almacenamiento y comunicaciones, y iv) agricultura, ganadería, pesca, caza y silvicultura, cuyas tasas de informalidad oscilan entre el $50 \%$ y el $70 \%$ - siendo más vulnerables el comercio, hoteles y restaurantes, y la construcción-; lo cual puede apreciar el lector en las figuras 6 y 7, donde se presenta el comportamiento de la informalidad.

Este tipo de actividades suelen demandar gran cantidad en mano de obra y poca especialización preparación educativa especialmente-, por lo cual los empleadores contratan estos individuos de manera informal, al no garantizar su afiliación al Sistema de Seguridad Social, y remunerarles por debajo del salario mínimo. Comercio, hoteles y restaurantes se ven afectados por la mayor tasa de informalidad, pues si una persona necesita desempeñarse en una actividad económica, las barreras de entrada y la inversión inicial para vender cualquier tipo de producto no son muy altas, es por esto que se observa la proliferación de vendedores ambulantes (Gómez, 2007). 


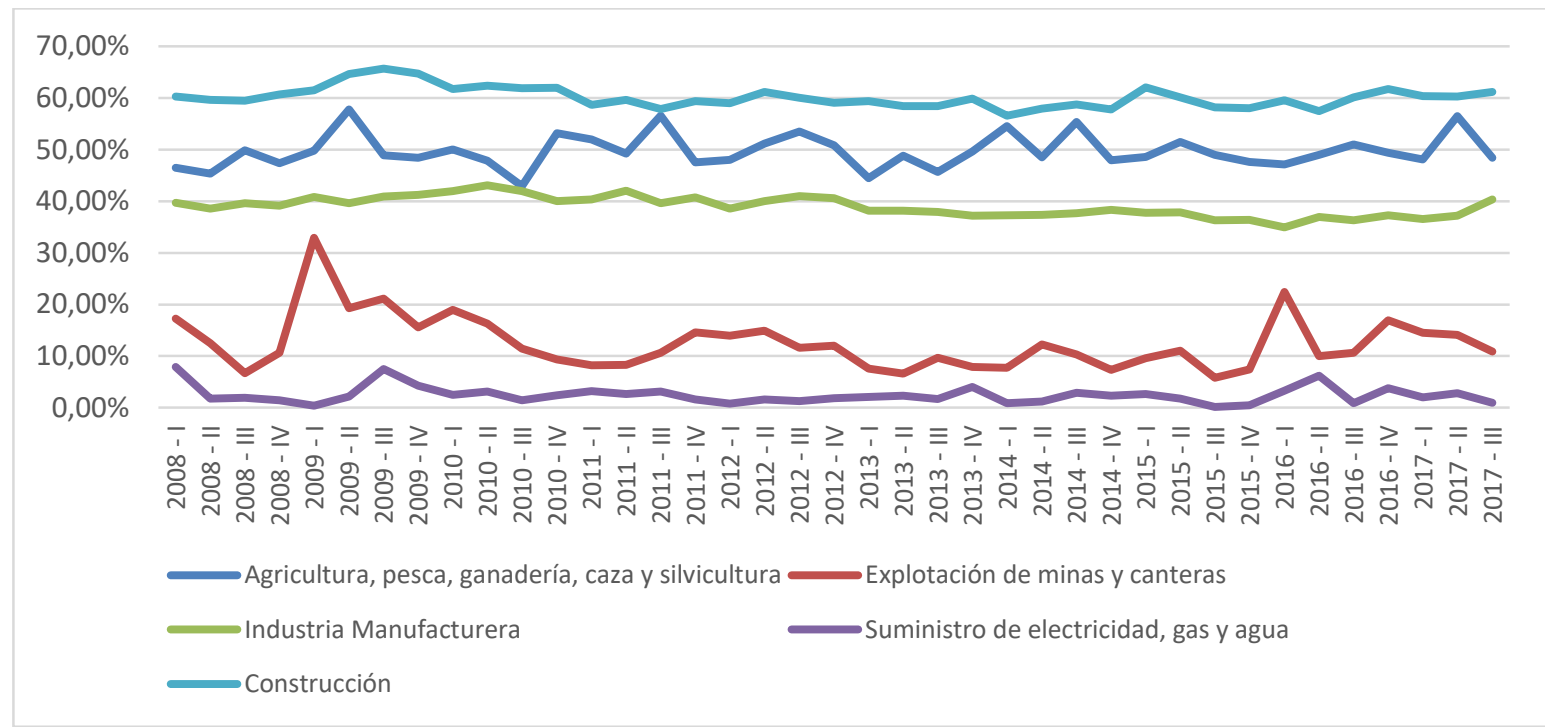

Figura 6. Tasa de informalidad por rama de actividad (1) en el período 2008 - 2017

Figure 6. Informal employment rate by sector (2008-2017)

Fuente: elaboración de los autores con datos de la Gran Encuesta Integrada de Hogares del DANE, 2017-2018)

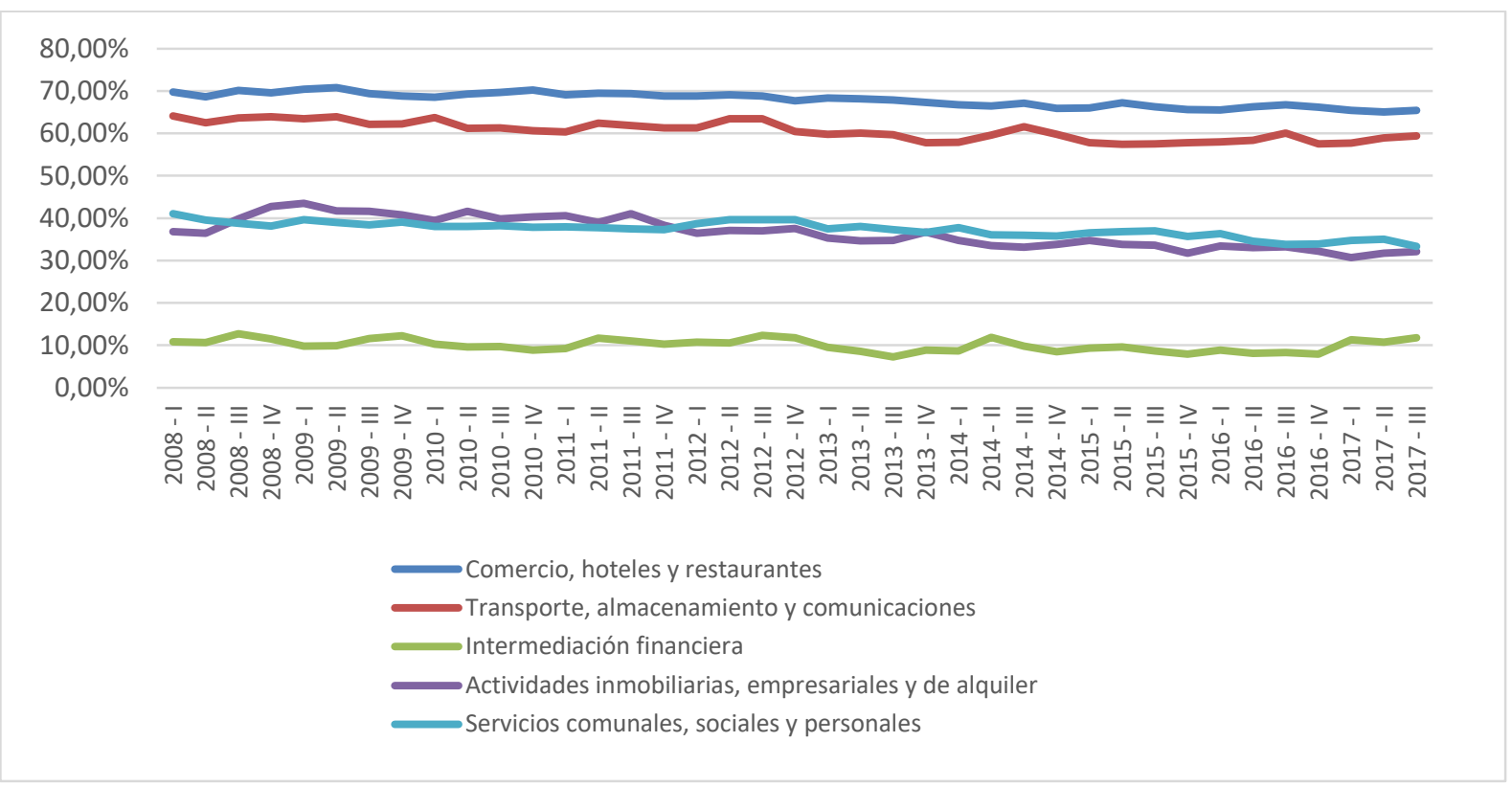

Figura 7. Tasa de informalidad por rama de actividad (2) en el período 2008 - 2017

Figure 7. (continued) Informal employment rate by sector (2008- 2017)

Fuente: elaboración de los autores con datos de la Gran Encuesta Integrada de Hogares del DANE, 2017-2018)

La informalidad en estos sectores debe su estado a una industria colombiana protegida por largas décadas que no logró un desarrollo sólido, fuerte y competitivo para enfrentar la globalización creciente y un modelo neoliberal, lo cual dio paso a un aumento en la tercerización; un sector agropecuario desprotegido, el cual está plagado de desigualdades en la distribución de la tierra, afectado totalmente por el conflicto interno y una incapacidad del Gobierno de realizar una reforma agraria efectiva que democratice la tierra. Lo anterior, genera que el sector sea improductivo y poco 
competitivo; aparte de esto, el Gobierno viene utilizando una política macroeconómica cuyo objetivo principal ha sido el control de la inflación y altas tasas de interés, que ha resultado en un detrimento en el aparato productivo y en efectos confusos en materia de crecimiento económico, control a la inflación, desempleo, alto costo del dinero y baja inversión, sobre todo en los sectores generadores de empleo como la industria y el agro (Gómez, 2007).

Estas situaciones generan un debilitamiento, reflejado en las altas tasas de informalidad de estos sectores; además, hacen que ramas más relacionadas con el sector terciario, como: las actividades inmobiliarias, empresariales y de alquiler; y la intermediación financiera, presenten tasas de informalidad más bajas, al ocurrir una migración de inversión al sector terciario.

\section{Ciudad}

De acuerdo con los datos obtenidos en el estudio realizado, Bogotá, Medellín, Manizales, Pereira y Cali, presentan las tasas de informalidad más bajas (entre $40 \%$ y $50 \%$ ), lo cual va vinculado con el hecho de que en estas ciudades (especialmente Bogotá, Medellín y Cali) se han concentrado los procesos de industrialización y emprendimiento más importantes de Colombia, además de ser ciudades con alta concentración demográfica, lo que permite que exista una importante demanda de bienes y servicios, generando oferta de empleo para satisfacer dichas necesidades; también, sobresale una fuerte presencia de las instituciones e infraestructura productiva que permiten altas tasas de ocupación formal, en contraste con ciudades como Cúcuta, Bucaramanga, Cartagena o Pasto (cuyas tasas de informalidad oscilan entre $55 \%$ y $70 \%$ ), las cuales se han caracterizado por estar relegadas al desarrollo experimentado en la región central del país; como consecuencia de esto, la cantidad de inversión extranjera que llega a esas ciudades no es la misma, y no hay un mercado tan fuerte. Por otra parte, en el caso de Bucaramanga, Cúcuta y Pasto, están ubicadas cerca de una frontera, por lo que el contrabando y las actividades transfronterizas pueden influir en la situación de informalidad.

En relación con lo expuesto, un estudio econométrico acerca de la informalidad laboral en los centros urbanos de Colombia concluye que al analizar la informalidad por ausencia de cotización a pensión, si el individuo reside en la región Atlántica o Pacífica, la probabilidad de ser desempleado o informal aumenta, mientras que la probabilidad de ser un trabajador formal disminuye; así mismo, cuando reside en ciudades de la región oriental del país el efecto marginal sobre la probabilidad de pertenecer al sector formal es positivo; por otro lado, este mismo efecto es negativo para explicar la pertenencia al sector informal; además, si el individuo reside en las regiones de Bogotá, Central, Antioquia, Valle o Archipiélago de San Andrés, la probabilidad de pertenecer al sector informal se reduce, mientras que la probabilidad de pertenecer al sector formal aumenta, dado que estas regiones presentan las mayores tasas de ocupación formal dentro de la muestra total (Romero, 2013); conjeturas que se articulan con la relación develada en este estudio, respecto a la informalidad y la ciudad de residencia.

\section{Costos de transacción y barreras de entrada}

Los costos de transacción y las barreras de entrada son dos aspectos relevantes para el estudio de la informalidad. Los costos de transacción aluden al coste que un individuo asume al realizar un movimiento económico, generado por fallas del mercado (Uribe, 2000); para este caso, se originan 
en intercambios económicos en el marco de la formalidad, de manera que se puedan contrastar con los generados en la informalidad.

Así, en condición de informalidad las personas jurídicas o naturales se ven obligadas a contribuir con el impuesto de industria y comercio, el cual es un gravamen directo que recae sobre la realización directa o indirecta de actividades industriales, comerciales o de servicios de manera permanente $u$ ocasional así sea con o sin establecimiento de comercio; además están en la obligación de llevar una contabilidad de sus actividades, siendo estos requerimientos los principales costos de transacción que enfrenta un individuo o empresa en condición de formalidad.

Romero (2013) realizó un estudio en Bucaramanga para determinar los costos de transacción exactos que afronta un informal si quiere formalizar su actividad; costos de oportunidad o ingresos sacrificados por interrupción de la actividad económica mientras se dispone de tiempo para realizar la gestión pertinente ante la entidad correspondiente; costos de movilización mínimos para desplazarse a la institución a fin de hacer las averiguaciones pertinentes o costo generado por la adquisición de servicio de internet para la consulta o realización del trámite; costo por tiempo de espera; el costo de completar un trámite; apoyo de un contador público que diligencie el libro fiscal de operaciones; y el impuesto a industria y comercio.

Por ello, es importante considerar que los costos anteriormente mencionados refieren únicamente a los requeridos para la legalización de la actividad comercial, siendo posible identificar otros costos de transacción asociados al ejercicio laboral de la actividad, tales como arriendo, servicios y gastos mínimos de manutención en el lugar de trabajo.

Igualmente, es de destacar que en condición de informalidad el individuo afronta principalmente los siguientes costos de transacción: costos relacionados por realizar transacciones únicamente en efectivo; costo generado por la vulnerabilidad de realizar su actividad en la calle; costos a largo plazo al no tener asegurada una vejez pensionada; costos de manutención; y los costos generados al no tener un ingreso estable. Otro posible costo que se da en algunas situaciones y más teniendo en cuenta el caso de Colombia, es el generado por el alquiler del puesto de trabajo como sucede en los centros de algunas ciudades. La diferencia de estos costos entre estos dos sectores (formal e informal) es posible denominarlos como los costos de transacción necesarios para formalizarse (Romero, 2013).

En cuanto a las barreras de entrada, para el contexto colombiano, cuando una persona quiere ingresar al sector formal, se enfrenta a barreras de entrada de tipo legal, las cuales son establecidas por la Cámara de Comercio, donde se incluyen aspectos como el registro único empresarial, registros en materia de tributación y otra serie de aspectos que permiten al empresario contar con los debidos respaldos normativos de industria y comercio (Romero, 2013); de allí que estos procesos impliquen asumir costos monetarios que contribuyen a la decisión del individuo de optar por la no formalización, al tener en cuenta los costos de transacción futuros que trae consigo la inserción al mercado formal.

De este modo, cuando se analiza un fenómeno como la informalidad, variables como las anteriormente expuestas deben ser consideradas, pues constituyen un factor que puede propiciar la decisión de no formalizarse, debido a los altos costos que tiene, en un contexto como el 
colombiano, la formalización de las actividades económicas; lo anterior, propone la necesidad de futuros estudios que exploren esta dimensión no sólo desde dimensiones cuantitativas, sino también con aproximaciones cualitativas.

\section{CONCLUSIONES}

La informalidad laboral es una problemática de creciente interés para los Gobiernos, no solo en función del daño que representa para los sistemas económicos, sino también por el impacto que posee en la calidad de vida de las personas que laboran bajo condiciones informales; por lo cual, los retos en materia de políticas públicas e intervenciones estatales son cada vez mayores.

Con la realización de este estudio, se develan determinantes cíclicos y acíclicos de la informalidad laboral en Colombia. Con respecto a los primeros -constituidos por el desempleo, la tasa de interés y la balanza comercial-, se infiere que existe una relación lineal directa entre la duración del desempleo y la informalidad; a mayor tiempo en calidad de desempleado, mayor probabilidad de ser informal. En cuanto a la tasa de interés, esta cumple un papel clave al ser una herramienta que facilita la circulación del dinero para realizar proyectos que fomentan la generación de empleo, crecimiento económico y mejores oportunidades para formalizar la actividad económica. Ahora, en relación con la balanza comercial, se refleja una brecha entre las importaciones y las exportaciones en el país, siendo mayor el número de importaciones, variable que afecta el fenómeno de desempleo, pues decrece la capacidad de las industrias para contratar mano de obra por la disminución de sus exportaciones; además, incrementan las probabilidades de que la población desocupada ingrese al mercado de la informalidad.

En cuanto a los determinantes acíclicos se pudo constatar que, al analizar la informalidad respecto al nivel educativo, se puede observar una influencia significativa entre esta variable y la informalidad, es decir, a mayor nivel educativo, menor probabilidad de pertenecer al sector informal. Frente a la rama de actividad donde el individuo desarrolle su actividad económica, existe una mayor probabilidad de informalidad en aquellas labores que requieren alta mano de obra, como el agropecuario, minero, construcción e industrial, y, por el contrario, menos informalidad en sectores terciarios como la intermediación financiera y la prestación de servicios públicos, lo cual se relaciona con los niveles de educación y las consecuencias ocasionadas por el conflicto interno.

La ciudad o área metropolitana influye en cierta manera sobre la economía informal; en el caso de Bogotá, Medellín, Cali y Manizales, se registraron las tasas de informalidad más bajas entre las 13 principales ciudades de Colombia, debido a la importancia y al desarrollo económico presente en estas regiones, además de que la inversión extranjera llega principalmente a estas ciudades y cuentan con mejor infraestructura productiva para ofrecer empleos de mayor calidad. En lo relativo a los costos de transacción y las barreras de entrada, el estudio permite develar una asociación, desde diversos postulados teóricos, entre estas variables y la informalidad laboral en el contexto colombiano, dado que las barreras de entrada al mercado formal son altas -especialmente desde perspectivas jurídicas-, lo que lleva a una mayor propensión a la informalidad.

Con este contexto, cabe resaltar la importancia de la identificación de estos determinantes, ya que a partir de esto es posible saber qué soluciones aplicar para resolver los problemas de informalidad 
causados por estas situaciones. Es claro que atacar todos los problemas al mismo tiempo puede ser un ejercicio infructuoso, al tener en cuenta la disponibilidad presupuestaria del Gobierno; es por eso que, para una mejora considerable, se deberían priorizar los recursos disponibles en asuntos como: i) apoyo a procesos de paz y reintegración de actores del conflicto armado a la sociedad, en virtud del impacto que este fenómeno ha tenido en el desplazamiento de la población y en su inserción en la informalidad, como alternativa de subsistencia al abandonar sus territorios de origen; y ii) promover una reforma tributaria que incentive el emprendimiento a baja escala, la cual permita remover carga tributaria a pequeñas y medianas empresas para que estas opten por formalizar sus actividades.

Como conclusión, es posible ampliar la investigación estructurando un modelo microeconómico en el que la informalidad esté en función de estos determinantes, para así plantear un modelo matemático. Adicionalmente, esto permitiría una regresión econométrica para el periodo expuesto, que permita observar cuál es el aporte puntual de cada uno de los determinantes a la tasa de informalidad anual, y si la variable dependiente muestral (tasa de informalidad), se acerca a la poblacional registrada por la GEIH.

\section{REFERENCIAS}

Arango, L. \& Flórez, L. (2017). Informalidad laboral y elementos para un salario mínimo diferencial por regiones en Colombia. Bogotá, Colombia: Banco de la República.

Banco Mundial (s.f.). Datos de libre acceso del Banco Mundial. Recuperado de https://datos.bancomundial.org/

Barco, D. \& Vargas, P. (2010). El perfil del trabajador informal y el retorno de la educación. Recuperado de http://www.bcrp.gob.pe/docs/Publicaciones/Documentos-deTrabajo/2010/Documento-de-Trabajo-04-2010.pdf

Barraza, N. (2010). Discriminación salarial y segregación laboral por género en las áreas metropolitanas de Barranquilla, Cartagena y Montería. Serie Documentos IEEC, 31(1), 1-44.

Berry, A. (2002). ¿Colombia encontró por fin una reforma agraria que funcione? Revista de Economía Institucional, 4(6), 24-70.

Carvajal, A., Cárdenas, L \& Estrada, I. (2017). Determinantes socioeconómicos de la informalidad laboral y el subempleo en el área metropolitana de Bucaramanga, Colombia. Equidad y Desarrollo, (29), 53-82. https://doi.org/10.19052/ed.4171

Casabón, C. (2017). La economía de América Latina supera por primera vez la de África Subsahariana. Recuperado de https://www.weforum.org/es/agenda/2017/05/la-economia-informal-deafrica-esta-retrocediendo-mas-rapido-que-la-economia-latinoamericana/

Castro, O. (2014). Informalidad y trabajo decente en Colombia: análisis desde la perspectiva de género. Relaciones Laborales y Derecho del Empleo, 2(3), 2 -17. 
Centro de Estudios del Trabajo. (2016). El rebusque en Colombia es el pan de cada día: la informalidad $y$ los TLC (2). Recuperado de http://cedetrabajo.org/wpcontent/uploads/2016/04/InformeNo2_alta_19abril.pdf

Consejo Privado de Competitividad. (2017). Informalidad, una nueva visión para Colombia. Bogotá, Colombia: Puntoaparte. Recuperado de https://compite.com.co/wpcontent/uploads/2017/11/CPC_Informalidad-V03.pdf

Departamento Administrativo Nacional de Estadística -DANE-. (2009). Metodología informalidad Gran Encuesta Integrada de Hogares - GEIH. Recuperado de https://www.dane.gov.co/files/investigaciones/boletines/ech/ech_informalidad/metodologia _informalidad.pdf

Departamento Administrativo Nacional de Estadística -DANE-. (2018). Colombia - Gran Encuesta Integrada de Hogares - GEIH 2018. Recuperado de http://microdatos.dane.gov.co/index.php/catalog/547/study-description

Galvis, L. (2012). Informalidad laboral en las áreas urbanas de Colombia. Coyuntura Económica: Investigación Económica y Social, 42(1), 15-51.

García, G. (2012). Determinantes macro y efectos locales de la informalidad laboral en Colombia. Sociedad y Economía, (21), 69- 98.

Gómez, L. G. (enero-junio, 2007). La informalidad en la economía, algo incuestionable. Semestre Económico, 10(19), 47-67. Recuperado de http://www.scielo.org.co/scielo.php?script=sci_arttext\&pid=S0120$63462007000100004 \& \operatorname{lng}=e n \&$ tIng=es

González, F. (2014). Poder y violencia en Colombia. Bogotá, Colombia: Editorial Pontificia Universidad Javeriana.

González, N \& Daza, N. (2015). Determinantes y perfiles de la participación laboral en Colombia en el periodo 2002-2013. Revista de Economía del Rosario, 18(1), 5-59. https://dx.doi.org/10.12804/rev.econ.rosario.18.01.2015.01

Jiménez, D. (2012). La informalidad laboral en América Latina: explicación estructuralista o institucionalista. Cuadernos de Economía, 31(58), 113-143.

Levy, S. y Székely, M. (2016). ¿Más escolaridad, menos informalidad? Un análisis de cohortes para México y América Latina. El trimestre económico, 83(332), 499-548. https://dx.doi.org/10.20430/ete.v83i332.232

Marcillo, E. (2010). ¿Existe alguna relación entre la informalidad laboral y la duración del desempleo? Un análisis exploratorio para Colombia (trece principales áreas metropolitanas, 2008). Recuperado de http://biblioteca.clacso.edu.ar/Colombia/cidseunivalle/20121113053738/DocTrab_133.pdf 
Mora, J. (2017). La informalidad laboral colombiana en los últimos años: análisis y perspectivas de política pública. Revista de métodos cuantitativos para la economía y la empresa, 24, 89-128.

Organización Internacional del Trabajo y Organización Mundial del Comercio. (2009). La globalización y el empleo informal en los países en desarrollo. Suiza: Organización Mundial del Comercio. Recuperado de https://www.wto.org/spanish/res_s/booksp_s/jobs_devel_countries_s.pdf

Osorio, L. (2016). Reforma tributaria e informalidad laboral en Colombia: Un análisis de equilibrio general dinámico y estocástico. Ensayos sobre Política Económica, 34(80), 126-145. https://dx.doi.org/10.1016/j.espe.2016.03.005

Palacios, M. (2012). Violencia pública en Colombia: 1958 - 2010. Bogotá, Colombia: Fondo de Cultura Económica.

Portes, A., y Haller. (2004). La economía informal. Recuperado de https://repositorio.cepal.org/bitstream/handle/11362/6091/1/S0411855_es.pdf

Quejada, R., Yánez, M \& Cano, K. (2014). Determinantes de la informalidad laboral: un análisis para Colombia. Investigación \& Desarrollo, 22(1), 126 - 145.

Ramírez, J., Zambrano, M., Mogrovejo, J \& Carreño, J. (2016). Informalidad laboral en los departamentos de Norte de Santander, La Guajira y Cesar. Revista Apuntes del CENES, 35(62), 125-145.

Rivas, A. y Sánchez, B. (2016). Políticas y resultados educativos en América Latina: un mapa comparado de siete países (2000-2015). Revista Electrónica de Investigación y Evaluación Educativa, 22(1), 1-30.

Romero, M. L. (2013). La informalidad como alternativa racional ante los costos de transacción. Un pequeño agujero puede hundir un barco: Una aproximación al caso de Bucaramanga Santander (trabajo de grado de pregrado). Bucaramanga, Colombia: Universidad Industrial de Santander.

Salinas, J., González, S \& Marín, L. (2012). Características de la población ocupada en Colombia: un análisis del perfil de los formales e informales. Perfil de Coyuntura Económica, (20), 57-86.

Simoes, A. \& Hidalgo, C. (2011). The Economic Complexity Observatory: An Analytical Tool for Understanding the Dynamics of Economic Development. Workshops at the Twenty-Fifth AAAL Conference on Artificial Intelligence. Recuperado de https://atlas.media.mit.edu/es/profile/country/col/

Uribe, E. M. (2000). La teoría de los costes de transacción y la fijación de los límites organizacionales al otro lado de las fronteras nacionales. INNOVAR, 1(16), 105-118. Recuperado de http://www.bdigital.unal.edu.co/26726/1/24375-85381-1-PB.pdf 
Véliz, J. \& Díaz, S. (2014). El fenómeno de la informalidad y su contribución al crecimiento económico: el caso de la ciudad de Guayaquil. Journal of Economics, Finance and Administrative Science, 19(37), 90-97. https://doi.org/10.1016/j.jefas.2014.09.001

Vergara, G., Ramírez, J., Naranjo, S., Carranza, J. (2017). Una estrategia de auto sostenibilidad de bajo costo para pasar de la economía informal a la economía formal. El caso de la Comuna 10 de Cali, Colombia. Revista Científica General José María Córdova, 15(19), 201-214. https://doi.org/10.21830/19006586.87 